\title{
Tietze's Syndrome in the emergency department: A rare etiology of atraumatic chest pain
}

\author{
Lee Grodin ${ }^{1 *}$, Gino Farina ${ }^{2}$ \\ ${ }^{1}$ Department of Emergency Medicine, State University of New York, Downstate Medical Center, New York, USA; \\ *Corresponding Author: Lee.Grodin@Downstate.edu \\ ${ }^{2}$ Department of Emergency Medicine, Long Island Jewish Medical Center, New York, USA
}

Received 29 March 2013; revised 30 April 2013; accepted 15 May 2013

Copyright ( 2013 Lee Grodin, Gino Farina. This is an open access article distributed under the Creative Commons Attribution License, which permits unrestricted use, distribution, and reproduction in any medium, provided the original work is properly cited.

\begin{abstract}
Tietze's Syndrome is an uncommon disorder that presents with painful, tender, non-suppurative swelling of the anterior chest wall. We report a case of a female patient who presented to the emergency department with a chief complaint of atraumatic chest pain and swelling of the anterior chest wall. After a thorough history and physical examination, as well as basic laboratory tests and chest radiography, she was diagnosed with Tietze's Syndrome. The expedient accurate diagnosis of Tietze's Syndrome is important for the physical and emotional well-being of a patient, and avoids overlooking more dangerous pathologies. Tietze's Syndrome needs to be considered in the differential diagnosis of a patient presenting with spontaneous swelling of the anterior chest.
\end{abstract}

Keywords: Tietze's Syndrome; Atraumatic Chest Pain

\section{INTRODUCTION}

Chest pain is a frequent chief complaint in emergency departments, and the underlying pathology ranges from completely benign to imminently life-threatening. Among the myriad possible etiologies are the confounding musculoskeletal disorders, which account for $50 \%$ of benign causes [1]. While frequently discussed and included in differential diagnoses, they are often inappropriately consolidated and misunderstood. Tietze's Syndrome (which has also been referred to as Costosternal Syndrome, Anterior Chest Wall Syndrome, Parasternal Chondrodynia, Thoracochondalgia, Chondropathia Tuberose, and Costochondral Junction Syndrome) is an uncommon musculoskeletal pathology, which was first described in 1921 by Alexander Tietze [2-4]. The National Institutes of Health lists it as a rare disease, indicating the limited available information about the syndrome as well as its possible rarity [5].

While it is a benign, self-limited, chronic pain condition characterized by a localized swelling involving usually the second or third ribs, unrecognized it can mimic malignancies, acute coronary syndrome, pneumonia, or trauma [6,7]. The emotional and physical relief of an accurate diagnosis is then delayed and the patient can undergo an emotionally stressful and costly evaluation for more serious disease $[7,8]$. The pain can be tremendous. We present a young female patient with a classic case of Tietze's Syndrome. The diagnostic difficulty lies in part to the absence of confirmatory laboratory or imaging tests, thus leaving the clinician reliant on the patient's history and presentation.

\section{CASE PRESENTATION}

A 29 years old otherwise healthy woman presented with localized right sided chest pain for approximately three days and focal swelling over the same area for one to two days. She described the pain as "aching," localized to the right side of her anterior chest, and radiating to her back ipsilaterally. It was pleuritic and associated with subjective shortness of breath, which she attributed to her pain. It was minimally responsive to ibuprofen. The swelling was very firm, tender, and had appeared spontaneously.

She denied trauma, recent travel, or illnesses. She denied fever, chills, malaise, or weight loss. She works as a visual merchandiser but has had no change in her level of physical activity.

One year ago, the patient reported she was diagnosed with Lyme Disease after presenting with malaise and Bell's Palsy. After confirmatory serological testing, she completed a course of antibiotics. At that time she also 
underwent evaluation for thyroid dysfunction and fibromylagia, neither of which was diagnostic and her symptoms were attributed to Lyme Disease. Her only surgery was approximately three years ago when she underwent a small bowel resection secondary to obstructive benign tumors.

On physical exam the patient preferred to be still in effort to minimize her pain. The only remarkable finding was a tender swelling approximately $5 \mathrm{~cm}$ in diameter over her right second and third costocartilages. There was no discoloration (ecchymosis or erythema) or other changes to the skin (Figure 1). The swelling was very firm and non-fluctuant. An electrocardiogram and chest radiograph revealed no abnormalities. Her basic lab work was within normal limits (CBC, CMP) and her ESR was $2.0 \mathrm{~mm} /$ Hour.

The diagnosis was discussed with the patient and her partner, and various pain management strategies were offered. In the emergency department she received ketorolac and warm compresses. Upon discharge she elected to continue ibuprofen, at increased doses, as well as use warm compresses. She was also prescribed oxycodone for break through pain.

During follow-up by telephone, the patient reported she declined to ever seek treatment or further imaging, and the pain persisted for two to three months, slowly declining. She has since returned to work and is no longer using any analgesics.

\section{DISCUSSION}

A timely diagnosis of Tietze's Syndrome can expedite the implementation of an effective treatment regiment, avoid an anxiety-provoking and costly work up, and reduce the possibility of overlooking more serious underlying pathologies. Tietze's Syndrome is rare and few clinicians have significant experience with it. The etiology is mysterious, however, its diagnostic profile has been crystallizing over the last half century.

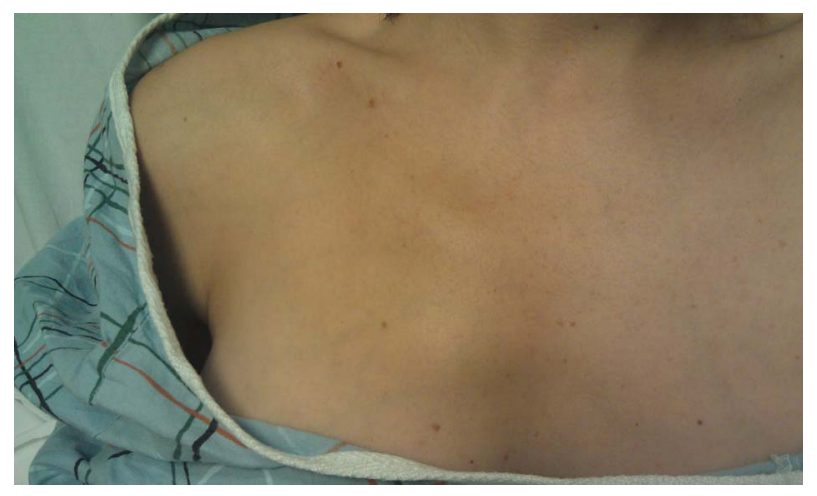

Figure 1. 29-year-old woman presenting with stereotypical localized swelling in Tietze's Syndrome on the anterior chest wall.
The incidence of Tietze's Syndrome is not well studied, but in 1976, a study of 320 patients with precordial chest pain yielded almost a $10 \%$ incidence of Costosternal Syndrome, including Tietze's Syndrome [3]. It is most commonly diagnosed in 20 to 50 year old patients, but can strike young pediatric and elderly patients $[6,9,10]$. There is a wide range of sex distribution reported, ranging from equal to twice as common in females than males $[6,7,11,12]$. The onset of pain can be sudden or gradual, and it is often pleuritic or aggravated my movement $[7,11,13]$. Supporting the diagnosis is the chronic, often relapsing and remitting course of Tietze's Syndrome and ruling out other possibilities by history, physical, and imaging [11,14].

The location of the pain and the rarity of the syndrome lay the foundation for a broad differential diagnosis. While $70 \%$ of cases of Tietze's Syndrome are unilateral and affect one joint, it is important to consider neoplastic, cardiac, respiratory, gastrointestinal, and other musculoskeletal origins [1]. While swelling is considered a hallmark of Tietze's Syndrome, it may only indicate the severity of disease [15]. Several cases of malignancy misdiagnosed as Tietze's Syndrome have underscored the importance of making an accurate diagnosis [16-22]. In addition one must be wary of the possibility of concurrent cardiac pathology [23].

In recent decades imaging has become an invaluable resource for clinicians, yet it is still not diagnostic of Tietze's Syndrome. While radiographs help minimize the concern for bone pathology, they are generally completely normal. Computer tomography scanning, bone scintigraphy, ultrasound, and magnetic resonance imaging have shown more helpful distinctions. A 2008 study recommends MRI and a 2010 review recommended bone scintigraphy and ultrasound as screening tests [11,15]. There have been some histological inquiries, but the studies have thus far deemed histology to be unrevealing or unimportant $[3,6,12]$.

Treatment for Tietze's Syndrome is supportive, with the mainstay of NSAIDs and application of heat or cold [13]. For refractory cases, corticosteroid injections may be considered [15].

\section{CONCLUSION}

Tietze's Syndrome is an idiopathic chronic pain condition, which is rarely seen in emergency departments. It presents with atraumatic chest pain and a focal swelling of the anterior chest wall. Current epidemiology suggests that its prevalence is highest among patients between 20 and 50 years of age. Accordingly it is important to consider Tietze's Syndrome and other musculoskeletal diagnoses in patients of this age range. Older patients presenting with chest pain are more likely cardiac, pulmonary, or neoplastic etiologies. The accurate diagnosis of 
Tietze's Syndrome provides the best platform for pain management, reducing the time and cost of an unnecessary work up for other conditions while not overlooking malignancies. It provides the patient with physical and emotional comfort. While a thorough history and physiccal are essential for diagnosis, follow up is helpful to ensure the disease course is consistent with Tietze's Syndrome and not suggestive of another pathology.

\section{REFERENCES}

[1] Gijsbers, E. and Knaap, S.F. (2011) Clinical presentation and chiropractic treatment of Tietze Syndrome: A 34-year-old female with left-sided chest pain. Journal of Chiropractic Medicine, 10, 60-63. doi:10.1016/j.jcm.2010.10.002

[2] Hiramuro-Shoji, F., Wirth, M.A. and Rockwood Jr., C. (2003) Atraumatic conditions of the sternoclavicular joint. Journal of Shoulder and Elbow Surgery, 12, 78-88. doi:10.1067/mse.2003.128566

[3] Wolf, E. and Stern, S. (1976) Costosternal Syndrome: Its frequency and importance in differential diagnosis of coronary heart disease. Archives of Internal Medicine, 136, 189-191. doi:10.1001/archinte.1976.03630020043010

[4] Tietze, A. (1921) Über eine eigenartige häufung von fällen mit dystrophie der rippenknorpel. Berliner Klinische Wochenschrift, 58, 829-831.

[5] Rare Diseases and Related Terms, National Institutes of Health (2013). http://rarediseases.info.nih.gov/RareDiseaseList.aspx?Pag $\mathrm{eID}=1$.

[6] Aeschlimann, A. and Kahn, M.F. (1990) Tietze's Syndrome: A critical review. Clinical and Experimental Rheumatology, 8, 407-412.

[7] Jelenko, C. and Cowan, G. (1977) Perichondritis (Tietze’s Syndrome) at the xiphisternal joint: A mimic of severe disease. Journal of the American College of Emergency Physicians, 6, 536-542. doi:10.1016/S0361-1124(77)80424-3

[8] Epstein, S.E., Gerber, L.H. and Borer, J.S. (1979) Chest wall syndrome: A common cause of unexplained cardiac pain. The Journal of the American Medical Association, 26, 2793-2797. doi:10.1001/jama.1979.03290520017017

[9] Mukamel, M., Kornreich L., Horev, G., Zebria, A. and Mimouni, M. (1997) Tietze's Syndrome in children and infants. Journal of Pediatrics, 131, 774-775. doi:10.1016/S0022-3476(97)70114-8

[10] Pikning, J.M., De Boeck, H. Desprechins, B. and Ernst, C. (2003) Tietze's Syndrome in a 2-year-old boy. Nederlands Tijdschrift Geneeskunde, 43, 2134-2136.
[11] Volterrani, L., et al. (2008) Magnetic resonance imagine in Tietze's Syndrome. Clinical and Experimental Rheumatology, 26, 848-853.

[12] Landon, J. and Malpas, J.S. (1959) Tietze’s Syndrome. Annals of Rheumatic Disease, 18, 249-254. doi:10.1136/ard.18.3.249

[13] Semble, E.L. and Wise, C.M. (1988) Chest pain: A rheumatologist's perspective. Southern Medical Journal, 81, 64-68. doi:10.1097/00007611-198801000-00014

[14] Langford, C.A. and Mandell, B.F. (2012) Chapter 336. Arthritis associated with systemic disease, and other arthritides. In: Longo, D.L., et al., Harrison's Principles of Internal Medicine, The MacGraw-Hill Companies, Inc., New York, 2858-2859.

[15] Stochkendahl, M.J. and Christensen, H.W. (2010) Chest pain in focal musculoskeletal disorders. Medical Clinics of North America, 94, 259-273. doi:10.1016/j.mcna.2010.01.007

[16] Fioravanti, A., Tofi, C., Volterrani, L. and Marcolongo, R. (2002) Malignant Lymphoma Presenting as Tietze's Syndrome. Arthritis and Rheumatism, 47, 229-230. doi:10.1002/art.10401

[17] Koren, W. and Shahar, A. (1998) Xiphodynia masking acute myocardial infarction: A diagnostic cul-de-sac. Journal of Emergency Medicine, 16, 177-178.

[18] Mathew, A.S., El-Haddad, G., Lilien, D.L. and Takalkar, A.M. (2008) Costosternal chondrodynia simulating recurrent breast cancer unveiled by FDG PET. Clinical Nuclear Medicine, 33, 330-332. doi:10.1097/RLU.0b013e31816a7a38

[19] Cocco, R., Galieni, P., Bellan, C. and Fioravanti, A. (1999) Lymphomas presenting as Tietze's Syndrome: A report of 4 clinical cases. Annali Italiani di Medicina Interna, 14, 118-123.

[20] Jeon, I.H., Jeong, W.J., Yi, J.H., Kim, H.J. and Park, I.H. (2010) Non-Hodgkin's lymphoma at the medial clavicular head mimicking Tietze's Syndrome. Rheumatology International, 32, 2531-2534. doi:10.1007/s00296-010-1397-2

[21] De Filippo, M. et al. (2007) MRI findings of Tietze's syndrome mimicking mediastinal malignancy on MDCT. European Journal of Radiology Extra, 65, 33-35. doi:10.1016/j.ejrex.2007.10.006

[22] Krohn-Gimberghe, B., Kurzeja, R., Wagner, J. and Kaiser, H.E. (1999) Pleomorphic T-cell lymphoma with chondropathia tuberose; A case report and review. Anticancer Research, 19, 2221-2228.

[23] Epstein, S.E., Gerber, L.H. and Borer, J.S. (1979) Chest wall syndrome: A common cause of unexplained cardiac pain. The Journal of the American Medical Association, 26, 2793-2797. doi:10.1001/jama.1979.03290520017017 